\title{
A Simplified method to identify patient face type for a prosthodontic treatment plan
}

\section{Introduction}

Biomechanical stress is an important risk factor in prosthetic dentistry. It has been demonstrated to be associated with occlusal wear, dentoalveolar trauma, as well as magnifying the risk of stress- related complications. Biomechanical stress intensity is directly related to force applied..$^{1-3}$ Many clinical studies have shown a correlation between bite force and facial structure. Facial morphology and jaw muscle size are important factors for the degree of bite force.$^{4-8}$ Facial type assessment is in many aspects crucial for the planning and prognosis of prosthetic treatment. Subjects with a strong bite force present a well-developed masticatory musculature, smaller anterior facial height (short face), and a smaller value in the gonial angle. They are called brachyfacial. Patients with smaller bite force value exhibit a longer anterior and shorter posterior facial height (long face) and a larger gonial angle. These patients are called dolichofacial. ${ }^{9-11}$ The terms brachyfacial, dolichofacial, and mesofacial, commonly used by orthodontists, were introduced in orthodontic literature by Ricketts RM. ${ }^{5}$ With regards to the effects of the occlusal load, scientific literature describes patients more prone to overload as individuals who present parafunctions. ${ }^{12-14}$ However, patients with parafunctions do not necessarily present load forces intense enough to cause damage to the periodontal tissues and teeth. On the contrary, brachyfacial individuals, even in the absence of parafunctions, develop considerably intense bite forces. These jeopardize the integrity of conservative and prosthetic rehabilitation and provoke a broad range of complications. ${ }^{15}$ Considering the face form of the patient in prosthodontic treatment planning may lead to a better clinical outcome if a relationship between face form and bite force is established. An individual bite force level can be determined with complex electronic devices. ${ }^{6}$ These instruments are not of easy use in the clinical practice and it is possible to use clinical alternative methods to recognize brachyfacial patients. The aim of the present study is to provide a diagnostic protocol that easily and safely allows recognizing high occlusal risk brachyfacial patients in order to implement a prosthetic therapy that is able to limit possible biological or mechanical damage caused by excessive bite forces.

\section{Clinical report}

The brachyfacial musculo-skeletal type patient is, muscularly, the strongest individual that the dentist can encounter and can be identified through a series of diagnostic investigations, more specifically three distinct exams: clinical, radiographic, and cephalometric.

\section{Clinical exam}

The clinical exam consists in observing the face of the patient both frontally and sagittally. In the frontal view, the patient presents a short lower face third, voluminous and tonic masseters and a square jaw. The dental arch is typically wide with a protruding mental symphysis. In general the face expresses sturdiness and strength (Figure 1). In the profile view, we can see a reduced nose-chin distance, developed masseter muscles, a wide mandibular base and the chin well represented with an often heavily pronounced sublabial

\author{
Volume 8 Issue 4 - 2017 \\ Luca Ortensi,' Marco Martini, ${ }^{2}$ Marco \\ Montanari, ${ }^{3}$ Gabriele Galassini ${ }^{3}$ \\ 'Department of Prosthodontics, University of Catania, Italy \\ ${ }^{2}$ Department of Maxillo Facial Surgery, University of Rome, Italy \\ ${ }^{3}$ Private practice, Italy
}

Correspondence: Luca Ortensi, Department of

Prosthodontics, University of Catania,Via De Gombruti, 540I23,

Bologna, Italy, Email lucaortensi I5@gmail.com

Received: October 05, 2017 | Published: October 10, 2017

sulcus. In the toothed patient it is possible to perform a simple clinical maneuver that can immediately reveal which musculo-skeletal class the individual belongs to. The dentist can place a ruler at the base of the mandible and see, from a sagittal view, if the imaginary extension of the instrument is tangent to the occipital bone or if it is lower. If the imaginary line is lower than the occipital bone, the patient can be classified as brachyfacial (Figure 2). This test have not to use singly, but always associated with other exams. This test cannot be applied to the edentulous patient and the dentist will have to base the diagnosis on parameters that will be described hereafter.

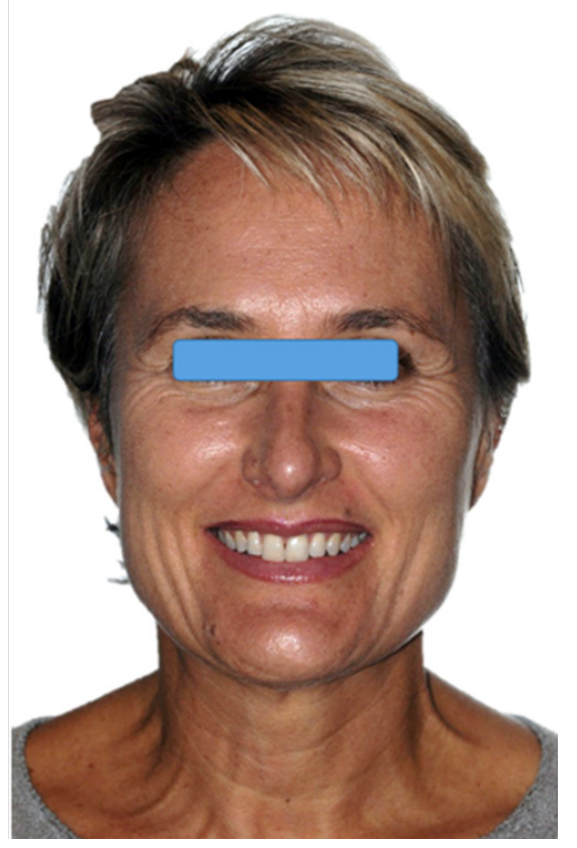

Figure I Brachyfacial patient (front view): short lower face third, voluminous and tonic masseters and a square jaw. 


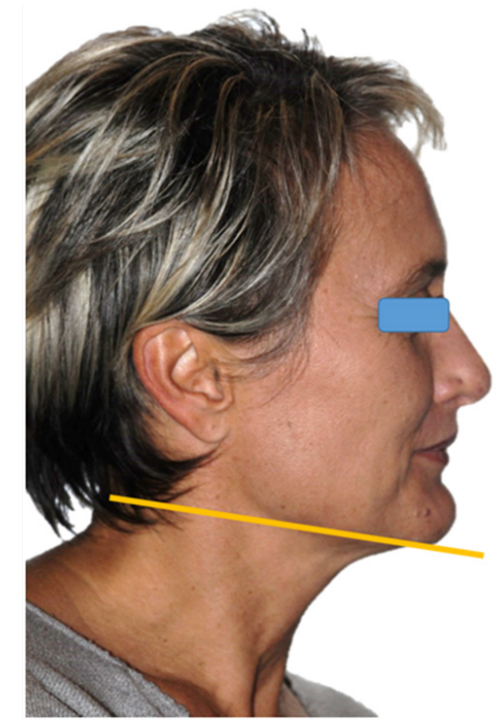

Figure 2 Brachyfacial patient (profile view): reduced nose-chin distance, developed masseter muscles and a wide mandibular base.

\section{Radiographic exam}

The preferred radiographic exam to identify different musculoskeletal types is latero-lateral radiography. The first author to introduce teleradiography in orthodontics was Broadbent BH. ${ }^{16}$ The lateral radiography has demonstrated to be an effective tool for use in the orthodontic and prosthetic plan of treatment. The use of this diagnostic medium makes it possible to assess some anatomical aspects even prior to performing actual cephalometric tracing: for example the geometric type, even before the numeric entity, of the mandibular angle. This angle is formed, simply speaking, by joining the mandible ramus with the mandibular base, which appears straight in the brachyfacial individual (Figure 3). Even in toothed patients latero-lateral radiography makes it possible to trace an ideal line tangent to the mandibular base, repeating what was clinically performed and described above (Figure $4 \& 5$ ).

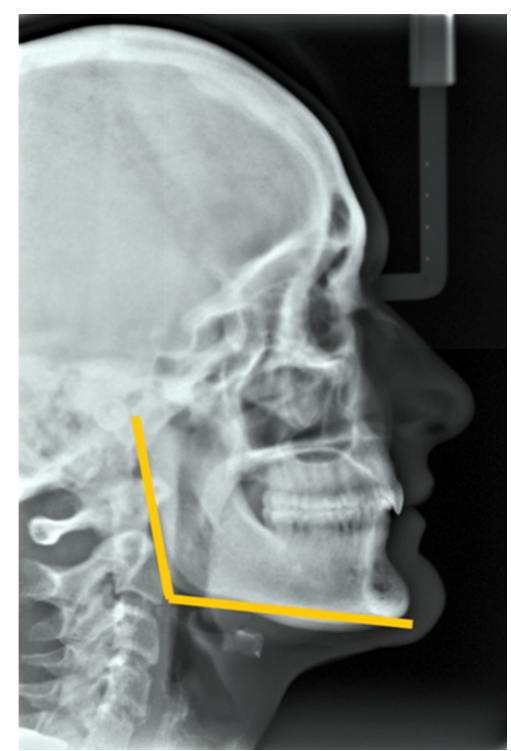

Figure 3 Latero-lateral $\mathrm{X}$-ray showing the mandibular angle, which is straight in the brachyfacial individual.

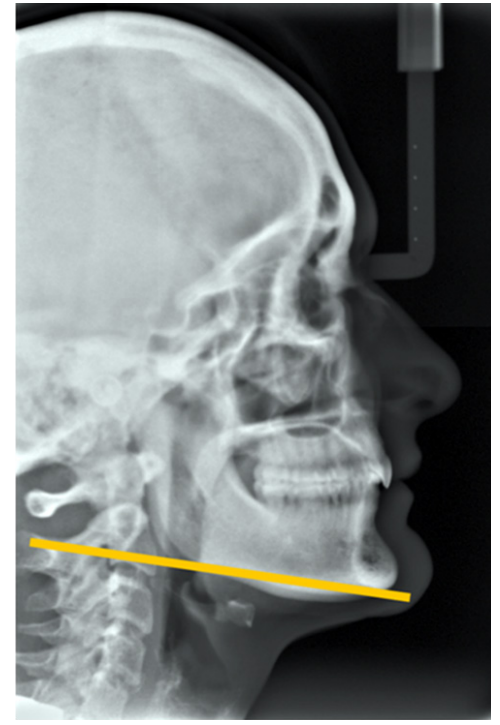

Figure 4 Latero-lateral $X$-ray showing the tangent drawn at the mandibular base which, in brachyfacial patients, starts below the occipital bone.

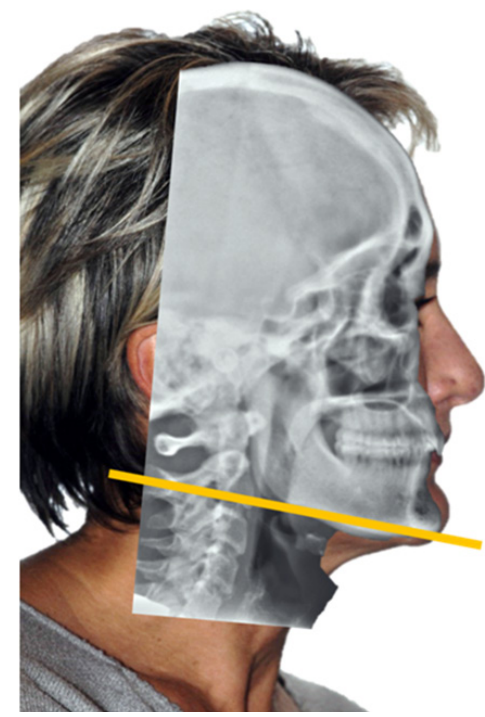

Figure 5 Superimposition (profile view) of the patient's photo and the laterolateral X-ray.

\section{Cephalometric exam}

A cephalometric analysis is without a doubt the most accurate diagnostic medium for recognizing a brachyfacial patient and many authors have suggested measuring the various angles in order to identify facial types. ${ }^{17-19}$ As this article is addressed to general dentists by acquiring just a few easily attainable points of analysis, it is possible to form a diagnosis that does not call for the complex and indispensable knowledge required by an orthodontic diagnosis. Below the most important points and angles for our simplified cephalometric analysis are provided. The gonial angle, that is the angle between the articular point (ar), the Gonion (Go) and the menton (me) is the angle that expresses the numeric entity of the angle formed by the base of the mandible and the mandible ramus. The normal value is $130^{\circ}+/-7$ : lower values identify a brachyfacial individual; higher values indicate a counterclockwise growth of the mandible, which 
therefore characterize the dolichofacial patient. ${ }^{4-18}$ Another method of analysis, for toothed subjects, can be to draw, on the latero-lateral $\mathrm{X}$-ray, the Frankfurt Plane (the line that joins the Porion and the Orbital Point) and the Mandibular Plane (the plane that is tangent to the lower edge of the mandibular body): the angle formed by the two is the Mandibular Plane Angle whose normal values at full growth are $23^{\circ}$ in males and $24^{\circ}$ in females $+/-4$. Values lower than the ones described identify a brachyfacial individual; higher values, indicate a dolichofacial individual (Figure 6) ${ }^{19-21}$ Visually, the more the two lines tend towards parallelism, the squarer the individual's mandible will be with a strong musculature and reduced vertical dimension, thus identifying it with the brachyfacial musculo-skeletal type; the more the two lines diverge, the thinner the mandible in the symphysis area will be, along with weak muscles and a long face, typical of dolichofacial individuals (Figure 7). As dentists becomes more familiar with analyzing latero-lateral X-rays, the skills and experience to easily identify which musculo-skeletal group an individual belongs to will be acquired. As mentioned previously, the edentulous individual with a brachyfacial musculoskeletal type is difficult to recognize and the dentist only has the cephalometric parameter available to identify it. In edentulous patients the diagnostic method to implement is that of measuring the gonial angle on the latero-lateral X-ray, since the value of the angle is independent of the presence or absence of teeth and other anatomical parameters such as: vertical dimension, mandibular position, mandible/cranial ratio and skeletal class (Figure 8). ${ }^{22}$

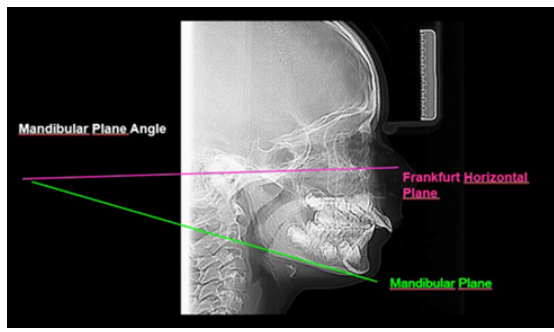

Figure $\mathbf{6}$ View of the Mandibular Plane angle formed by joining the Frankfurt Plane and the Mandibular Plane.

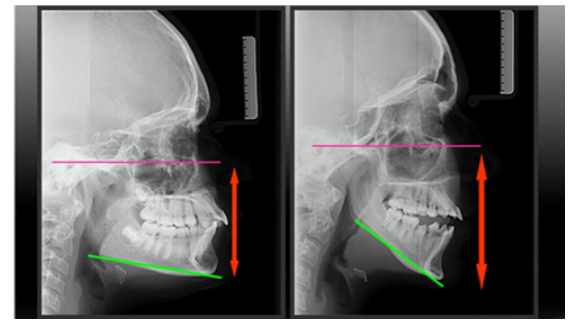

Figure 7 The lines that form the mandibular angle in the various face types: left, brachyfacial individual; right, dolichofacial individual.

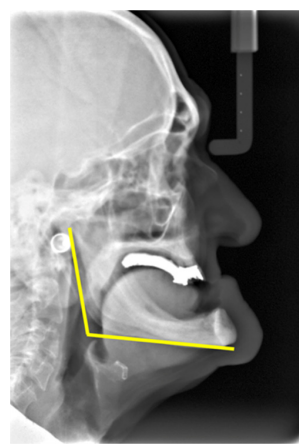

Figure $\mathbf{8}$ The edentulous individual with brachyfacial musculo-skeletal type is more difficult to identify: the diagnostic method to implement is to measure the gonial angle on the latero-lateral X-ray.

\section{Discussion}

Brachyfacial individuals have been broadly studied in literature both for aspects of anatomical interest and for the proven muscular force expressed during chewing cycles that makes them "particular patients." The clinical implications that can be deduced from this scientific knowledge have been vastly applied in orthodontics with a change of therapeutic protocols and the diversification of treatment plan based on musculoskeletal type. ${ }^{23}$ For other dental specializations, in particular for prosthodontics and implantology, this did not occur if only as a generic recommendation, by some authors, ${ }^{24}$ to be aware of these patients. As mentioned, literature often associates occlusal risks to the presence or absence of parafunctions, which should be evaluated when formulating a treatment plan. However, their effects will vary depending on the type of patient suffering from them. For example, a brachyfacial individual who clenches his/her teeth will apply to the dentition - whether natural or artificial - joint structures pressure forces that are three or four times greater than a dolichofacial individual. ${ }^{8}$ In brachyfacial individual the parafunction is an aggravating circumstance of the occlusal risk and not the risk itself. As evidence shows, general dentists, prosthetists, and implantologists must take the shape of the patient's face into account, similarly to other diagnostic elements when planning a more or less complex therapy. Additionally, a protocol must be put into place that reduces possible overload complications to a minimum. As mentioned earlier, in the presence of a natural or artificial dentition, clinical and radiographic tests can be sufficient to identify brachyfacial patients. In edentulous subjects and in case of diagnostic doubt, the cephalometric exam becomes crucial for a correct diagnosis. Even thought this test is not easily performed, the simplification of the cephalometric test proposed, with the mere purpose of identifying square-face individuals, can easily become applicable by all dentists and not just orthodontic specialists.

\section{Conclusion}

In both simple and complex clinical cases, an occlusal load is a necessary risk factor to take into account when formulating a treatment plan. We are aware that brachyfacial patients present a considerable bite force regardless of the presence or absence of parafunctions. The objective was to provide a diagnostic protocol that simple and safely, allows recognizing these individuals and easily implementing an odontological therapy - in particular prosthetic and implanting treatment - that can limit possible damage caused by excessive bite force. Further clinical studies are needed to validate this promising protocol.

\section{Acknowledgments}

The authors thank Dr. Maria Laura Strocchi for her assistance in preparing this manuscript.

\section{Conflicts of interest}

The Author declares that there is no conflict of interest.

\section{Funding}

None.

\section{References}

1. Kiliaridis S, Johansson A, Haraldson T, et al. Craniofacial morphology, occlusal traits, and bite force in persons with advanced occlusal tooth wear. American Journal of Orthodontics and Dentofacial Orthopedics. 1995;107(3):286-292. 
2. Helkimo E, Carlsson GE, Carmeli Y. Bite force in patients with functional disturbances of the masticatory system. Journal of Oral Rehabilitation. 1975;2(4):397-406.

3. Johansson A. A cross-cultural study of occlusal tooth wear. Swed Dent J Suppl. 1992;86:1-59.

4. Shinogaya, T, Bakke M, Thomsen CE, et al. Effects of ethnicity, gender and age on clenching force and load distribution. Clinical Oral Investigations. 2001;5(1):63-68.

5. Ricketts RM. A foundation for cephalometric communication. American Journal of Orthodontics. 1960;46(5):330-357.

6. Koc D, Dogan A, Bek B. Bite force and influential factors on bite force measurements: a literature review. Eur J Dent. 2010;4(2):223-232.

7. Bakke M. Bite force and occlusion. Seminars in orthodontics. 2006;12(2):120-126.

8. Braun S, Hans-Peter, William P Hant, et al. A study of bite force, part 1 Relationship to various physical characteristics. The Angle Orthodontist. 1995;65(5):367-372.

9. Boyd SB, Gonyea WJ, Finn RA, et al. Histochemical study of the masseter muscle in patients with vertical maxillary excess. J Oral Maxillofac Surg. 1984;42(2):75-83.

10. Pereira LJ, Gavião M, Bonjardim LR, et al. Muscle thickness, bite force, and craniofacial dimensions in adolescents with signs and symptoms of temporomandibular dysfunction. The European Journal of Orthodontics. 2007;29(1):72-78.

11. Farella M, Bakke M, Michelotti A, et al. Masseter thickness, endurance and exercise-induced pain in subjects with different vertical cranio-facial morphology. Eur J Oral Sci. 2003;111(3):183-188.

12. De Boever JA, Carlsson GE, Klineberg IJ. Need for occlusal therapy and prosthodontic treatment in the management of temporomandibular disorders. Part I. Occlusal interferences and occlusal adjustment. J Oral Rehabil. 2000;27(5):367-379.
13. Lobbezo F, Van Der Zaag J, Naeije M. Bruxism: its multiple causes and its effects on dental implants-an updated review. J Oral Rehabil. 2006;33(4):293-300.

14. Isidor F. Influence of forces on peri-implant bone. Clinical Oral Implants Research. 2016;17 Suppl 2:8-18.

15. Lasilla V, Holmlund I, Koivumaa KK. Bite force and its correlations in different denture types. Acta Odontol Scand. 1985;43(3):127-132.

16. Broadbent BH. A new X-ray technique and its application to orthodontia: the introduction of cephalometric radiography. The Angle Orthodontist. 1981;51(2):93-114.

17. Ricketts RM. Planning treatment on the basis of the facial pattern and an estimate of its growth. The Angle Orthodontist. 1957;27(1):14-37.

18. Ricketts RM. A foundation for cephalometric communication. American journal of Orthodontics. 1964;46(5):330-357.

19. Schudy FF. Vertical growth versus anteroposterior growth as related to function and treatment. Angle Orthod. 1964;34(2):75-93.

20. Bishara SE, Ortho D, Jakobsen JR. Longitudinal changes in three normal facial types. Am J Orthod. 1985;88(6):466-502.

21. Ingervall, B, Minder C. Correlation between maximum bite force and facial morphology in children. Angle orthod. 1997;67(6):415-424.

22. Uppal S, Gupta NK, Tandan A, et al. Comparative evaluation of vertical dimension at rest before extraction, after extraction and after rehabilitation with complete denture-A Cephalometric study. J Oral Biol Craniofac Res. 2013;3(2):373-377.

23. Pepicelli A, Woods M, Briggs C. The mandibular muscles and their importance in orthodontics: a contemporary review. American Journal of Orthodontics and Dentofacial Orthopedics. 2005;128(6):774-780.

24. Misch CE. Contemporary implant dentistry. $3^{\text {rd }}$ ed. USA: Mosby-Elsevier Health Sciences; 2013. 1120 p. 